113 f.), plädiert gegen eine schwarz-grüne Koalition (S. 198), schilt die Länder, dass sie sich nicht für einen lebendigen Föderalismus stark machen (S. 241 ff.), wirbt nicht nur für die Neuordnung des Länderfinanzausgleichs, sondern auch für eine Länderneugliederung, deren Kommen er sogar wegen haushaltspolitischer Notwendigkeiten voraussagt (S. 243), und unterstützt den Kurs Merkels in der Euro-Krise (S. 277 - 295). Auch liefert Stoiber am Schluss seines Buches einen kurzen Besinnungsaufsatz zur „Zukunft der Demokratie“ (S. 297 - 319), in dem er unter anderem - man hört und staunt - für die Einführung von Volksentscheiden auf Bundesebene eintritt. All dies zeigt, dass sich der 71-Jährige noch viel zu jung fühlt für den politischen Ruhestand, in den er 2007 gezwungen wurde. Immerhin wurde ihm die Chance eröffnet, sich in Brüssel ehrenamtlich für den Abbau der EU-Bürokratie einzusetzen - was er nicht nur nach eigenem Bekunden (vgl. S. 260 - 267) recht erfolgreich tut.

Die vergleichende Betrachtung der drei Leben führt zu einem beruhigenden Schluss: Die Bundesrepublik Deutschland verfügt über einige Politiker, die das Zeug zum Kanzler haben. Nicht alle (sogar die wenigsten), die Kanzler können, werden es auch. Doch selbst als verhinderte Kanzler machen sie sich weiter nützlich - was Steinbrück gegebenenfalls noch beweisen könnte. Nach dem zurückliegenden Wahlkampf hätte er sich aber auch den Ruhestand mehr als verdient.

Patrick Horst

\title{
Chronist und Protokollant deutscher Außenpolitik: ein aufschlussreiches Tagebuch
}

\author{
Majonica, Ernst: Das politische Tagebuch 1958-1972, bearbeitet von Hans-Otto Kleinmann \\ und Christopher Beckmann (Forschungen und Quellen zur Zeitgeschichte, Bd. 55), Droste \\ Verlag, Düsseldorf 2011, 765 Seiten, € 69,-.
}

Wem sagt heute der Name Ernst Majonica noch etwas? Selbst bei Zeithistorikern scheint er weitgehend in Vergessenheit geraten zu sein. Majonica gehörte von 1950 bis 1972 dem Deutschen Bundestag an und war über viele Jahre profilierter und prominenter Außenpolitiker der Unionsfraktion, deren außenpolitischer Sprecher und Arbeitskreisvorsitzender er über mehr als zwei Wahlperioden bis 1969 war.

$\mathrm{Zu}$ seinem umfangreichen publizistischen Werk - Majonica verfasste in seiner Abgeordnetenzeit allein vier Bücher (unter anderem 1965 den Band „Deutsche Außenpolitik“) gehört sein Tagebuch, das er von 1952 bis 1982 kontinuierlich führte und in dem er Ereignisse, Aktivitäten und Begegnungen notierte. Es handelt sich bei den komplexen Aufzeichnungen „um den seltenen Fall eines Ego-Dokuments aus der ersten Parlamentariergeneration der Bundesrepublik“, so die Bearbeiter. Die vorliegende Auswahl aus dem Tagebuch ist eine „die politischen Inhalte zusammenführende Komposition der Bearbeiter“, ein Quellenauszug der Jahre 1958 (Majonicas Wahl zum Geschäftsführer des Arbeitskreises V für auswärtige, gesamtdeutsche und Verteidigungsfragen der CDU/CSU-Fraktion) bis zu seinem Ausscheiden aus dem Bundestag 1972. Die von Majonica eigentlich nicht zur Veröffentlichung vorgesehenen Tagebuchnotizen ermöglichen einen Einblick in das „Innenleben" von Parlaments- und Parteigremien jenseits offizieller Protokolle und dokumentieren 
den außenpolitischen Diskurs der Unionsfraktion von der zweiten Berlinkrise bis zur Auseinandersetzung um die Ostverträge aus der subjektiven Sicht eines außenpolitisch versierten und eigenständigen Abgeordneten. Dem Leser gibt das Tagebuch daneben einen Einblick in das aufreibende Tagesgeschäft eines umtriebigen und engagierten Parlamentariers und Parteipolitikers zwischen Ausschussarbeit, Parteigremiensitzungen, Wahlkreisauftritten und Wahlkämpfen.

Majonica wurde 1920 im westfälischen Soest in kleinbürgerlichen Verhältnissen geboren und starb hier 1997 im Alter von knapp 77 Jahren. Der überzeugte Katholik studierte in Münster und Freiburg Jura. Bereits die Wahl seiner Vorlesungen zeigt ausgeprägte historische und philosophisch-theologische Interessen. Gegen die NS-Ideologie immunisierte ihn die Verwurzelung im katholischen Milieu. Seine juristische Ausbildung konnte Majonica nach Kriegsdienst und Gefangenschaft fortsetzen. Sein Vater gehörte in Soest zu den Mitgründern der CDU, so kam er zur Partei. 1950 gelang dem jungen Assessor und kurzzeitig praktizierenden Rechtsanwalt der entscheidende Einstieg in die Bundespolitik: Auf dem Goslarer Gründungsparteitag der Bundes-CDU wurde Majonica zum Bundesvorsitzenden der Jungen Union gewählt. Er behielt dieses Amt bis 1955, als er eine Kampfabstimmung gegen den acht Jahre jüngeren Gerhard Stoltenberg aus Schleswig-Holstein verlor. Sein Bundestagsmandat erlangte Majonica erst im „zweiten Anlauf“ im November 1950, als nach dem Mandatsverzicht des nordrhein-westfälischen Landesministers und späteren Bundespräsidenten Heinrich Lübke im Wahlkreis Arnsberg/Soest eine Nachwahl fällig wurde (bis 1953 fand für ausscheidende direkt gewählte Abgeordnete ein Nachwahl statt). Den Wahlkreis gewann Majonica in fünf Wahlen jeweils mit beachtlichen Mehrheiten, sicherlich wegen seiner starken parlamentarischen und medialen Präsenz und seinen unablässigen Aktivitäten im Wahlkreis. Anfänglich war er das jüngste Fraktionsmitglied. In der zweiten Wahlperiode entwickelte sich Majonica zum außenpolitischen Experten seiner Fraktion und wurde 1959 als Nachfolger von Kurt Georg Kiesinger Vorsitzender des außenpolitischen Arbeitskreises. In diesem Amt war er für zehn Jahre eine gewichtige, eigenständige außenpolitische Stimme der Union im Bundestag. Bald entwickelte er auch ein besonderes Interesse für Asien. Obwohl anfangs ein großer Bewunderer Konrad Adenauers lehnte Majonica die gaullistische Europa-Politik und Adenauers Nähe zu Charles de-Gaulle vehement ab und bezeichnete den französischen Staatspräsidenten in seinem Tagebuch als „Antieuropäer Nr. 1“. Bei der Ratifizierung des Élysée-Vertrages half er mit, die „atlantische“ Präambel durchzusetzen.

Auf dem Höhepunkt seiner Politikerlaufbahn galt Majonica zwar als ministrabel, blieb aber bei den Regierungsbildungen 1965 und 1966 unberücksichtigt. Seinem Tagebuch vertraute er das Gefühl des Scheiterns an und litt darunter, vom eigentlichen Gestalten der Politik ausgeschlossen und lediglich ein „Kommentator des Zeitgeschehens“ zu sein. In den eigenen Reihen geriet er zunehmend in eine Außenseiterposition und wurde schließlich 1969 als Arbeitskreisvorsitzender abgewählt und von den „Falken“ in der Fraktion in den Hintergrund gedrängt. Das zeigte sich nicht zuletzt in der heftigen Diskussion um die Ostpolitik der Regierung Brandt/Scheel. Der Realpolitiker Majonica fürchtete im Falle ihres Scheiterns nachteilige Auswirkungen auf die Zusammenarbeit mit den atlantischen Partnern. Die Politik der CDU bereitete ihm zunehmend „Unbehagen“. Sein politisch-parlamentarischer Stern begann zu sinken, in seiner Fraktion war er zunehmend isoliert. Bei den vorgezogenen Bundestagswahlen des Jahres 1972 wurde der gerade 52jährige von seiner Partei in seinem Soester Wahlkreis, den er 22 Jahre als direkt gewählter Abgeordneter im Bundestag vertreten hatte, nicht mehr nominiert. 
Sein allmählicher Rückzug aus der praktischen Politik, auch in seiner Landespartei, ging einher mit einer intensiveren politikwissenschaftlichen Beschäftigung: 1971 wurde Majonica an der Universität München mit einer Arbeit über die Beziehungen zwischen der Bundesrepublik Deutschland und der Volksrepublik China zwischen 1949 und 1966 promoviert. Kurz vor dem Ende seiner politischen Karriere kamen dabei seine lebenslange Beschäftigung mit der Geschichte und Kultur Asiens zum Ausdruck, aber auch seine Stärke in der theoretischen Analyse und sein überwiegend wissenschaftlich-historisches Herangehen, das in seinem außenpolitischen Denken überwog.

Nicht zuletzt die detaillierten Anmerkungen und die ausführliche Einleitung der Bearbeiter machen die vorliegende Tagebuchedition zu einem gut und mit Gewinn lesbaren Stück allgemeiner Geschichte der Außenpolitik Deutschlands und besonders der ersten beiden Jahrzehnte der Bundesrepublik.

Joachim Rott

\section{Politische Repräsentation: Krise, Wandel, Reform?}

Linden, Markus und Winfried Thaa (Hrsg.): Krise und Reform politischer Repräsentation, Nomos Verlagsgesellschaft, Baden-Baden 2011, €29,-.

Alonso, Sonja, John Keane und Wolfgang Merkel (Hrsg.): The Future of Representative Democracy, Cambridge University Press, Cambridge 2011, € 27,40.

Je stärker sich Gesellschaften funktional differenzieren und dabei nationalstaatliche Grenzen relativieren, desto größer werden die normativen und praktischen Herausforderungen, die mit dem zum Teil krisenhaft wahrgenommenen Wandel repräsentativer Demokratie einhergehen. In den letzten Jahren haben sich zwei Tagungsprojekte an der Universität Trier und am Wissenschaftszentrum Berlin für Sozialforschung (WZB) diesen theoretischen wie empirischen Herausforderungen gestellt und sich mit dem Status Quo sowie den Wandlungstendenzen demokratischer Repräsentation befasst. Aus diesen Auseinandersetzungen sind zwei Sammelbände hervorgegangen, die zusammengenommen einen breiten Einblick in die aktuelle Repräsentationsforschung geben. Beiden Publikationen liegt ein ähnliches Repräsentationsverständnis zugrunde: Sie wird als eine erhaltenswerte Form der Organisation demokratischer Herrschaft verstanden, die historischen Wandlungsprozessen unterworfen ist. Vor allem die Pluralisierung der Repräsentanten und der Repräsentationsbeziehungen ist ein alle Beiträge durchziehendes Thema.

Der von Markus Linden und Winfried Thaa herausgegebene Sammelband geht auf eine Tagung des Teilprojekts C 7 „Formen und Funktionsweisen politischer Repräsentation von Fremden und Armen in der Bundesrepublik Deutschland“ des SFB 600 „Fremdheit und Armut“ 2010 in Trier zurück und fragt nach Krisendiagnosen und Reformmöglichkeiten politischer Repräsentation. Die Herausgeber führen in ihrer Einleitung fünf typische Argumentationsweisen an, mit denen die Krise politischer Repräsentation beschrieben wird: Es gäbe (1) eine systematische Benachteiligung allgemeiner Interessen beziehungsweise Anliegen, und zwar (2) sowohl bei der Vermittlung als auch Durchsetzung; (3) eine zunehmenden Privatisierung und Entfremdung zwischen Staat und Bürger, (4) die zu einer Legi- 